\title{
Gradient Strengthening Control Procedure Based on Numerical Simulation of Combined Local Loading Of Deformation Zone
}

\author{
Olga V. Pilipenko \\ Orel State University (OSU) \\ Orel, Russian Federation \\ Vyacheslav A. Golenkov \\ Orel State University (OSU) \\ Orel, Russian Federation
}

\begin{abstract}
A gradient strengthening control procedure based on the numerical simulation of the combined local loading of the deformation zone in the form of an algorithm of actions pertinent to the prescription of operational parameters is described. A particular case in the form of the completed nomogram of the treatment parameters prescription for obtaining of strengthening predetermined by the depth and value is presented.
\end{abstract}

Keywords-plastic metal working, combined local loading of the deformation zone, numerical simulation, industrial process control, Odkvist parameter, microhardness, strengthening.

\section{INTRODUCTION}

Nowadays, in order to increase the operational life of the machines, different processing methods are used, one of which is based on changing the mechanical characteristics of the outer layers of contacting parts. Such methods are usually referred to as strengthening ones. These methods may differ by their basic physics and, therefore, may include both phase transformations and changes in the chemical composition, as well as the fragmentation of the structural constituents. One of the key problems of such processes is obtaining the required quantitative target characteristics, particularly, the hardness (microhardness) of the surface layers and strengthening depth. Hence the crucial task includes the control of these processes.

However, for most existing processes, the solution of this problem often cannot be fulfilled in practice and requires a vast scope of experimental surveys, particularly in case of approbation of new materials and alloys. Fewer difficulties arise if the strengthening process is based on the plastic metal working methods, particularly, on new methods involving the combined local loading of the deformation zone (hereinafter referred to as the "CLL deformation").

CLL deformation processes are defined as such processes in which the plastic deformation zone is created via applying two or more loads, one of which exerts an effect on the substantial volume of the piece, while another one bears a local character and creates a mobile deformation zone characterized by a combined stress state [1-11]. In practice, in order to obtain hollow axisymmetric products characterized by the gradient

\author{
Sergey Yu. Radchenko \\ Orel State University (OSU) \\ Orel, Russian Federation \\ Daniil O. Dorohov \\ Orel State University (OSU) \\ Orel, Russian Federation
}

strengthening spreading from the outer surface, the method may be realized following the scheme, which is presented in Fig. 1.

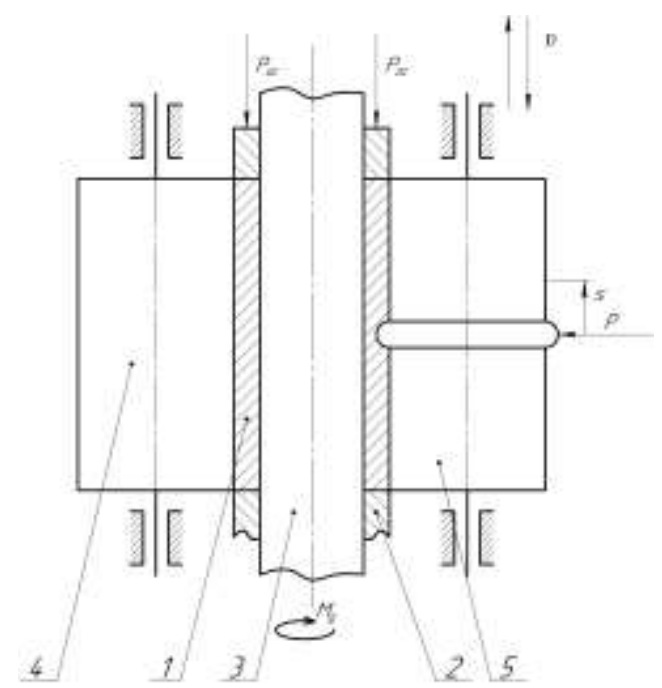

Fig. 1. Scheme illustrating the strengthening CLL deformation process: $1-$ piece; 2 - rests; 3 - mandrel; 4 - smooth roller (smoothing tool); 5 - burnisher (forming tool); $\mathrm{Mg}$ - moment applied to the piece; $\mathrm{v}$ - moving velocity of the burnisher; $\mathrm{P}$ - the force applied to the burnisher, which determines the penetration depth; Poc - the force applied to the rests, $\mathrm{S}-$ the feed pitch

The piece, in this case, is represented by collar 1 (see Fig. 1). The local deformation tools are represented by smooth rollers 4 and burnisher (the roller designed with special protrusion) 5 . The piece driving is realized from rests 2 via the rigid geometrical constraint; the global loading by force $P_{o c}$ is also transferred via the rests.

Gradient strengthening is realized from the outer surface, while the internal surface of the piece is supported by mandrel 3. This method of treatment allows obtaining the axisymmetric products, particularly metallic collars, characterized by the high strength properties, and provides the essentially new quality gradual (gradient) changing of hardness from the maximum value at the outer surface of the product up to the initial one. 
The methods of the strengthening CLL deformation due to the unique stress-strain state within the deformation zone allow treating difficult-to-form and nonplastic metals and alloys. The result of strengthening in case of CLL deformation depends on several parameters, the most important of which are the loading force, the feed pitch, the number of passages and the shape of the forming tool. Prescription and selection of these parameters for the considered treatment method determine the resulting strengthening.

The effectiveness of the controlled creation of gradient strengthened structures using CLL deformation methods is based on the proved quadratic distribution of microhardness values along the depth of the strengthened layer [12-14]. Thus, in the strengthening depth - microhardness coordinates, strengthening distribution is described by the parabolic curve, the vertex of which corresponds to the point of transfer from the strengthened structure to the unstrengthened one and determines the maximum strengthening depth. Eventually, the problem of controlling the strengthening CLL deformation processes is reduced to determining the effect of the loading force, the feed pitch, the number of passages and the shape of the forming tools on two principal parameters, which are the value and depth of strengthening; this approach substantially simplifies the development of the design methods pertinent to such processes.

\section{INVESTIGATION}

Previously, the works pertinent to the numerical mathematical simulation of CLL deformation following the method shown in Fig. 1 were performed and based on the analysis of the results of these works [15-20], a general algorithm of designing such processes for axisymmetric products, which allows realizing the gradient strengthening characterized by the predetermined characteristics, was proposed (Fig. 2).

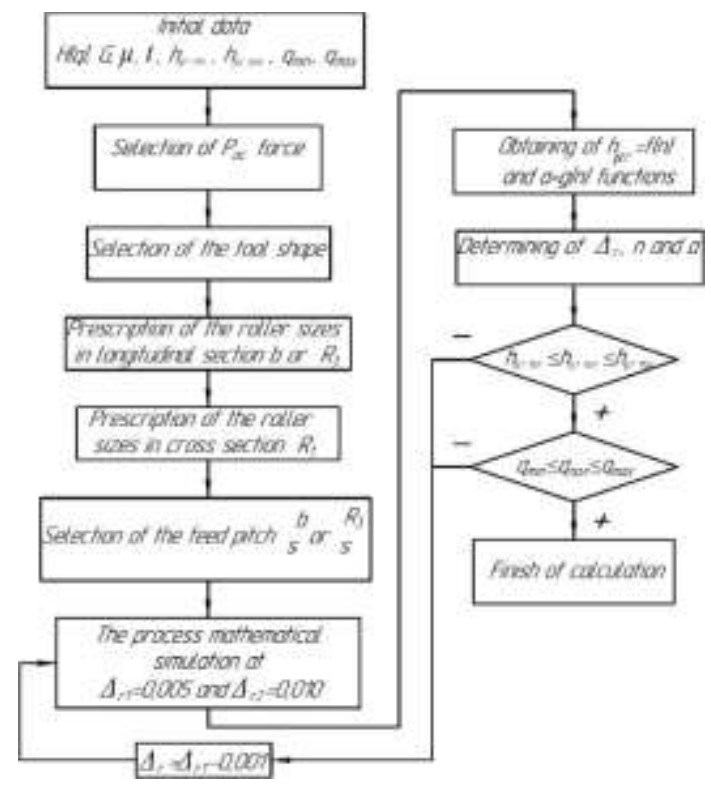

Fig. 2. General algorithm applied for designing metal working processes involving the combined local loading of the deformation zone, which allows realizing the controlled development of gradient strengthened structures in metals and alloys.
This algorithm was obtained based on the numerical analysis of the mathematical model of the process and it allows designing CLL deformation processes for the controlled development of the prescribed gradient strengthened structures in products.

At the initial stage, target strengthening characteristics $q_{\min }$ and $q_{\max }$, which correspond to the minimum and maximum values of the required Odkvist parameter in the surface layers, respectively, as well as $h_{\mu r \min }$ and $h_{\mu r \max }$, which correspond to the minimum and maximum values of the relative strengthening depth, are prescribed. In this case, the Odkvist parameter is connected with microhardness via the following equation:

$$
q=\ln \left(\sqrt[n]{\frac{k \cdot\left(\delta H_{\mu}\right)^{2}}{A}}+1\right)
$$

where $\mathrm{n}$ and $\mathrm{k} / \mathrm{A}$ - coefficients, which are reflecting the mechanical properties of the material;

$$
\delta H_{\mu} \text { - required microhardness gain. }
$$

The values of the coefficients $\mathrm{n}$ and $\mathrm{k} / \mathrm{A}$ in the equation (1) may be obtained from the results of the experiment, which involves the compression or tension of two similar specimens with different deformation ratios $\varepsilon$ and subsequent measuring of their hardness:

$$
\begin{gathered}
n=\ln \left(\frac{H_{\mu 1}-H_{\mu 0}}{H_{\mu 2}-H_{\mu 0}}\right) / \ln \left(\frac{\varepsilon_{1}}{\varepsilon_{2}}\right) \\
\frac{k}{A}=\frac{\left.\varepsilon_{1}^{\ln \left(\frac{H_{\mu 1}-H}{H_{\mu 2}-H}\right)}\right) / \ln \left(\frac{\varepsilon_{1}}{\varepsilon_{2}}\right)}{H_{\mu 1}-H_{\mu 0}}
\end{gathered}
$$

where $H_{\mu 1}$ and $H_{\mu 2} \quad-$ specimens hardness after compression or tension; $\varepsilon_{1}$ and $\varepsilon_{2}$-deformation ratios of the specimens.

At this stage, the mechanical characteristics of the piece are also prescribed, i.e. $\mathrm{H}(\mathrm{q})$ - the hardening curve, $\mathrm{G}$ - shear modulus, $\mu$ - coefficient of friction between the piece material and the tool; and $\mathrm{t}$ - thickness of the strengthened product.

At the second stage, the axial compression force is prescribed, while it should be taken into account that treatment involving applying the axial force with $p_{o c}>0.9 \cdot \sigma_{0}$ (where $\sigma_{0}$ - yield strength of the material of the treated piece) is twice as efficient compared with the scheme of treatment without axial compression. However, the implementation of such additional axial compression is expedient only in serial production, since in this case application of additional non-adjustable facilities will be required.

At the subsequent stages, the shapes and sizes of rollers are prescribed. For the strengthening CLL deformation processes, the following recommendations pertinent to selecting the shape 
of indenters (rollers) may be given. The roller with the cylindrical protrusion should be used in cases when high values of surface hardness and large strengthening depth are required. However, there is a risk of the surface damage in consequence of the availability of a large zone of shear tension stresses. The roller with the protrusion angle of $150^{\circ}$ should be used in cases when it is necessary to create a structure with the large strengthening depth and the assured small damage of the surface layers of the product (excessive cold-work hardening). However, the strengthening degree and gradient will be small (this type of the tool exerts a substantial influence on the strengthened layer depth, while the strengthening value is changed slightly). The roller with the toroidal protrusion allows realizing high values of surface hardness with a relatively large strengthening gradient. However, the energy consumption will be less than in case of the cylindrical roller and the zone characterized by the tension shear stresses will be small. The sizes of the forming tools may be prescribed using the following equations. For the roller with the toroidal protrusion:

$5.06 \cdot\left(R_{3}-\frac{\Delta_{r} \cdot t}{2}\right)>R_{1}>\frac{\Delta_{r} \cdot t \cdot R_{2}}{2 \cdot \mu \cdot R_{2}-\Delta_{r} \cdot t}$

For the roller with the cylindrical protrusion:

$1.125 \cdot \frac{b^{2}}{\Delta_{r} \cdot t}>R_{1}>\frac{\Delta_{r} \cdot t \cdot R_{2}}{2 \cdot \mu \cdot R_{2}-\Delta_{r} \cdot t}$

For the roller with the protrusion angle in the range of $140^{\circ}-150^{\circ}$, the upper boundary may be recommended as:

$$
R_{1}=12.65 \cdot \Delta_{r} \cdot t
$$

Explanations to the values, introduced into equations (4) and (5), are presented in Fig. 3.
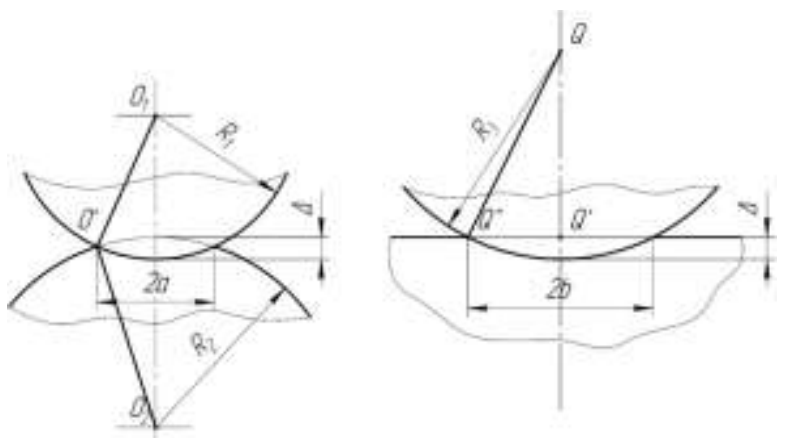

Fig. 3. Projections of the contact area in the cross and longitudinal sections: $\Delta$ - depth of the tool penetration into the piece body $(\Delta \mathrm{r}-$ relative penetration depth); R1 - tool radius in cross section; R2 - piece radius (outer) in cross section; R3 - tool radius in longitudinal section; $2 \mathrm{~b}$ - width of the cylindrical roller in the longitudinal section.

For selecting feed pitch $\mathrm{s}$, the following recommendations may be given:

- for the roller with the toroidal protrusion:

$$
0.5 \cdot R_{3} \leq s \leq R_{3}
$$

- for the roller with the cylindrical protrusion:

$$
0.33 \cdot b \leq s \leq b \text {. }
$$

The next stage of design involves mathematical simulation of the process for two values of the relative penetration depth, which is actually equivalent to the calculation performed for two different loading forces.

Based on the results of the mathematical simulation, functions $h_{\mu r}=f(n)$ and $a=g(n)$ are obtained. These functions are graphically represented in Figs. 4 and 5.

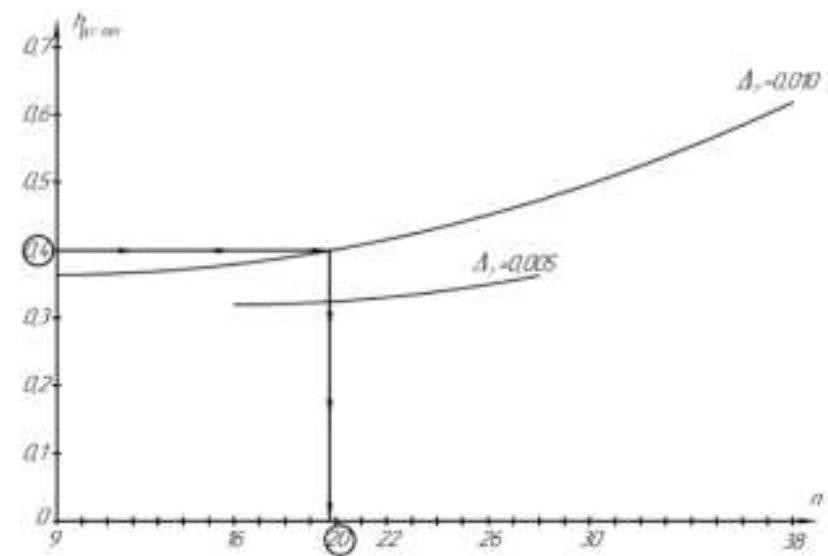

Fig. 4. Nomogram used for determining the number of passages in the course of strengthening CLL deformation. The value of the overlapping coefficient $\mathrm{b} / \mathrm{s}$ $=3.00$.

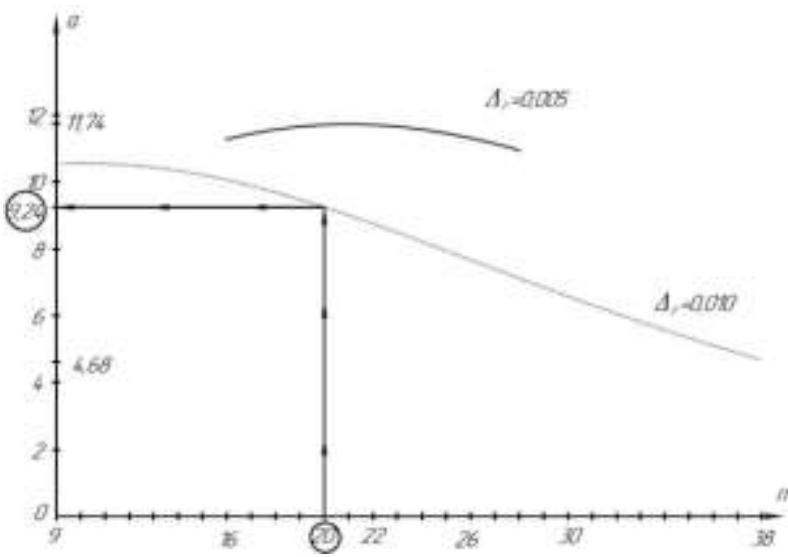

Fig. 5. Nomogram used for determining principal process parameter a in the course of strengthening CLL deformation. The value of the overlapping coefficient is $\mathrm{b} / \mathrm{s}=3.00$.

Then, after prescribing the necessary strengthening depth, the value of the relative penetration depth of the roller and the number of passages are determined. If the target value of the strengthening depth will not be obtained, then recalculation should be performed for the less penetration depth of the forming tool, and after obtaining satisfactory results, the calculation of the Odkvist parameter may be fulfilled using the following equation:

$$
q=\ln \left(\sqrt[n]{\frac{k \cdot a \cdot\left(h_{\mu r} \cdot t\right)^{2}}{A}}+1\right)
$$

If the value obtained using the equation (9) will not correspond to the interval estimate of the target characteristic 
pertinent to the surface strengthening, then another value of the load force (relative penetration depth) should be prescribed.

For the simplification of the process parameters prescription, aimed at the practical realization of strengthening via CLL deformation methods based on general technique (refer to Fig. 2), individual nomograms may be plotted (refer to the example presented in Fig. 6).

\section{CONCLUSION}

In this paper, based on the analysis of the results of the numerical simulation, a general algorithm of designing the metal working processes via the strengthening CLL deformation was proposed. The developed method made it possible to efficiently control the characteristics of the obtained gradient strengthening, i.e. via varying the process parameters (the loading force, the feed pitch, the number of passages and the shape of the forming tool) reliably to realize the target strengthening characteristics - the hardness of the surface layer and strengthening depth.

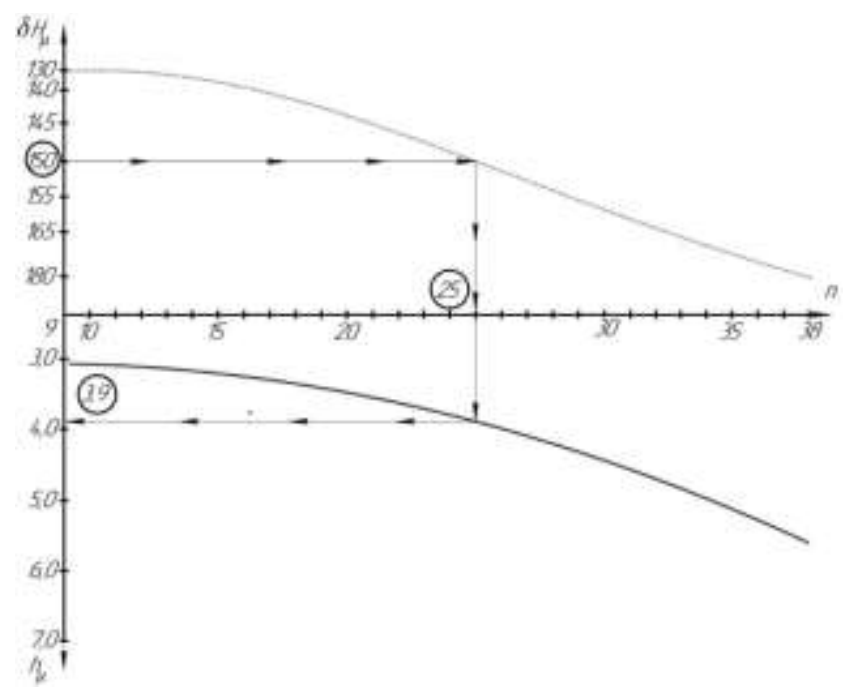

Fig. 6. Nomogram used for determining the process parameters in the course of strengthening CLL deformation for the particular case of $\mathrm{t}=10 \mathrm{~mm}$; the material - Cu90Sn5Zn5Pb5, forming a roller with the cylindrical protrusion. The outer diameter of the piece is equal to $45 \mathrm{~mm}$. The value of the overlapping coefficient is $\mathrm{b} / \mathrm{s}=3.00$, the value of the relative penetration depth is $\Delta \mathrm{r}=0.010$.

This paper was prepared under the project of "Engineering center of digital medium processes providing integrated safety and security: telecommunications, communication facilities and energy efficiency" within the tender on granting of the public support for the projects pertinent to foundation and development of engineering centers based on higher education institutions, subject to the general jurisdiction of the Ministry of Education and Science of the Russian Federation.

\section{References}

[1] V.A. Golenkov, S.Yu. Radchenko, D.O. Dorokhov, and G.P. Korotkii, "Scientific Foundations of Strengthening via the Combined Local Deformation", Moscow: Mashinostroenie, Oryol: State University UNPK, 2013.

[2] V.A. Golenkov, S.Yu. Radchenko, D.O. Dorokhov, and I.M. Gryadunov, "Classification of the combined local deformation processes",
Fundamental and Applied Problems of Engineering and Technology, vol. 6, pp. 85-89, 2010.

[3] G.D. Del, "Determination of Tension in Plastic Area on Distribution of Hardness", Moscow: Mashinostroenie, 1971

[4] A.V. Tretyakov and V.I. Zyuzin, "Mechanical Properties of Metals and Alloys When Forming", Moscow: Metallurgiya, 1973.

[5] G.E. Arkulis, "Theory of Plasticity: Manual for Higher Education Institutions", Moscow: Metallurgiya, 1987.

[6] O.L. Tolokonnikov, A.A. Markin, and V.F. Astapov, "Investigation of the Forming Process with the Finiteness of Deformation Taken into Account", Applied Mechanics, vol. 19, vol. 10, pp. 122-125, 1983.

[7] A.M. Abrão, B. Denkena, B. Breidenstein, and T. Mörke, "Surface and subsurface alterations induced by deep rolling of hardened AISI 1060 steel", Production Engineering Research and Development, vol. 8, vol. 5, pp. 551-558, 2014. http://dx.doi.org/10.1007/s11740-014-0539-x

[8] A. Akkurt, "Comparison of roller burnishing and other methods of finishing treatment of the surface of openings in parts from tool steel d3 for cold forming", Metal Science and Heat Treatment, vol. 53, pp. 145150, 2011. http://dx.doi.org/10.1007/s11041-011-9358-2

[9] S. Bagheri and M. Guagliano, "Review of shot peening processes to obtain nanocrystalline surfaces in metal alloys", Surface Engineering, vol. 25 , no. 1, pp. 3-14, 2009. http://dx.doi.org/10.1179/026708408X334087

[10] H. Hamadache, Z. Zemouri, L. Laouar, and S. Dominiak, "Improvement of surface conditions of $36 \mathrm{Cr}$ Ni Mo 6 steel by ball burnishing process", Journal of Mechanical Science and Technology, vol. 28, no. 4, pp. 1491 1498, 2014. http://dx.doi.org/10.1007/s12206-014-0135-1

[11] I.M. Gryadunov, V.A. Golenkov, O.V. Pilipenko, and S.J. Radchenko, "Hardening treatment by plastic deformation under conditions of the integrated local loading of a deformation zone", International Journal of Applied Engineering Research, vol. 12, no. 21, pp. 11094-11100, 2017.

[12] S.Yu. Radchenko, D.O. Dorokhov, and I.M. Gryadunov, "Evolution of BrOCS 5-5-5 alloy microstructure and mechanical parameters under intensive plastic deformation conditions", Solid State Phenomena, vol. 265 SSP, pp. 157-165, 2017.

[13] V.A. Golenkov, S.Y. Radchenko, D.O. Dorohov, and I.M. Gryadunov, "Microhardness distribution in the cross-section in case of strain hardening under combined local load", International Journal of Applied Engineering Research, vol. 11, no. 20, pp. 10315-10320, 2016.

[14] S. Radchenko, D. Dorohov, and I. Gryadunov, "The volumetric surface hardening of hollow axisymmetric parts by roll stamping method", Journal of Chemical Technology and Metallurgy, vol. 50, no. 1, pp. 104$112,2015$.

[15] O.V. Pilipenko, S.Yu. Radchenko, D.O. Dorokhov, and I.M. Gryadunov, "Connection of Odkvist parameter and values of microhardness when hardening by plastic deformation", International Journal of Applied Engineering Research, vol. 12, no. 13, pp. 3639-3644, 2017.

[16] P.G. Morrev, "A variational statement of quasistatic "rigid-deformable" contact problems at large strain involving generalized forces and friction," Acta Mechanica, no. 222, pp. 115-130, 2011. http://dx.doi.org/10.1007/s00707-011-0516-9

[17] P.G. Morrev and T.V. Fedorov, "A nurbs approximation of experimental stress-strain curves", Journal of Chemical Technology and Metallurgy, vol. 51, no. 3, pp. 341-349, 2016.

[18] T.V. Fyodorov, "Approximation of the experimental strengthening curves using non-uniform linear fractional B-splines", Fundamental and Applied Problems of Engineering and Technology, no. 1(303), pp. 64-68, 2014.

[19] P.G. Morrev and V.A. Gordon, "Simulation of surface hardening in the deep rolling process by means of an axial symmetric nodal averaged finite element", Journal of Physics: Conference Series, vol. 973, no. 1, 012013 , 2018

[20] P.G. Morrev and V.A. Gordon, "An axisymmetric nodal averaged finite element", Latin American Journal of Solids and Structures, vol. 15, no. 2. 